\title{
Differential outcomes from magneto- and electroencephalography for the analysis of human cognition
}

\author{
Carsten Eulitz ${ }^{\mathrm{a}, \mathrm{b}, *}$, Hind Eulitz $^{\mathrm{a}}$, Thomas Elbert ${ }^{\mathrm{a}}$

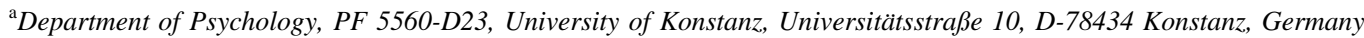 \\ ${ }^{\mathrm{b}}$ Institute for Experimental Audiology, University of Münster, Münster, Germany
}

Received 20 March 1997; revised version received 30 April 1997; accepted 2 May 1997

\begin{abstract}
Theoretical considerations show that magnetoencephalography (MEG) and electroencephalography (EEG) provide different information about ongoing human brain activity. The paper presents simultaneously measured MEG and EEG data showing that these measures may lead to different conclusions about cognitive models under investigation. This was demonstrated for amplitude results of the P300/N400 complex in a study of the secondary processing of lexical and non-verbal information in visual stimuli. As both methods provide different information about ongoing brain activity, their combined analysis is valuable. This seems particularly true for studies of higher order cognitive processing. (C) 1997 Elsevier Science Ireland Ltd.
\end{abstract}

Keywords: Magnetoencephalography (MEG); Electroencephalography (EEG); Event-related magnetic field; Evoked potential; Visual evoked activity

The same cognitive process may be differentially imaged by magnetoencephalography (MEG) and electroencephalography (EEG). The purpose of this paper is to quantitatively illustrate this point by using data from an experiment designed to investigate visual pattern recognition and processing. While differences between MEG and EEG may be less prominent for exogenous components of the eventrelated brain activity $[2,8]$, the dissociation may become pronounced for endogenous components reflecting higherorder processes.

Already from a theoretical point of view, such differences become plausible. EEG results from the extracellular volume currents triggered mainly by postsynaptic potentials. MEG is thought to arise from the intracellular branch, of this process, i.e. from the currents that flow from the dendritic tree to the soma. Thereby, MEG is mainly sensitive to currents flowing tangentially to the surface of the scalp [9] and to a lesser degree, about $10 \%$, to radial sources [6]. As a consequence EEG and MEG are affected differ-

\footnotetext{
* Corresponding author. Tel.: +49 7531 884260; fax: +49 7531882891 ; e-mail: carsten.eulitz@uni-konstanz.de
}

ently by averaging due to a stronger reduction of 'biological noise' for tangential sources than for radial ones.

Sources in the primary and secondary sensory projection areas as the Brodman areas 3b (somatosensory), 41/42 (auditory) or 17 (visual) are primarily tangentially oriented and are consistently activated in each trial. Consequently, for such sources activated early in the information processing, the signal-to-noise ratio is considerably higher for MEG measurements than for EEG measurements.

When higher processing stages are investigated, the corresponding sources may become more distributed [4], and currents flowing simultaneously in opposing walls of a sulcus may partially cancel each other. The remaining equivalent current dipole may have a stronger radial than tangential orientation [7] and thus will appear with a relatively greater weight in EEG than MEG responses. Therefore, complementary information such as EEG should be used in addition to MEG when brain activations beyond the primary and secondary projection areas are being studied. The present paper is aimed to support these theoretical considerations through experimental evidence. It will be demonstrated that the interpretation of MEG data in isolation may lead to different conclusions that cannot be main- 
tained if EEG data are also taken into consideration, and vice versa.

Experimental data were extracted from a combined MEG and EEG study of the higher order processing of visually presented information (for methods see [3]). The processing of lexical information through visually presented words was compared to three other experimental conditions requiring the processing of non-verbal information. The four different classes of visual stimuli were: (1) 'word' stimuli, content words with two syllables; (2) 'false font' stimuli, pattern of four rotated, mirrored or distorted letters; (3) 'shapes' stimuli, complex pattern with a row of four or five clusters of connected pixels; (4) 'dots' stimuli, randomly arranged pixels on an area identical to the other classes of stimuli. Visual stimuli were presented for $400 \mathrm{~ms}$. The interstimulus interval varied randomly from 2.0 to $2.4 \mathrm{~s}$. The primary task for the 13 healthy, right handed subjects was a target detection. The probability of a target to appear was $15 \%$ for all stimulus conditions. The experiment consisted of six blocks of 194 trials each, resulting in a total of 252 stimuli per experimental condition.

Neuromagnetic data were recorded using a 37-channel neuromagnetometer (BTi). The sensor array was centered over the left supra temporal cortex about $1.5 \mathrm{~cm}$ superior to the T3 electrode position of the 10-20 system. EEG data were recorded using a Neuroscan amplifier from the electrode positions F3, F4, T3, T4, P3, P4, M1 and M2 of the 10-20 system with $\mathrm{Cz}$ used as a reference. Horizontal and vertical electrooculogram and the button press was also recorded. Continuous data were recorded in 7-8 min blocks at a sampling rate of $297.6 \mathrm{~Hz}$ with a passband of $0.03-100$ $\mathrm{Hz}$. Averaged waveforms for non-targets were calculated for each stimulus class across epochs of $1500 \mathrm{~ms}$, including a $300 \mathrm{~ms}$ pre-stimulus baseline using artifact-free epochs, and were digitally filtered using a low-pass filter of $20 \mathrm{~Hz}$.

For the present paper the mean amplitude in the latency interval from 350-550 ms was evaluated. Several comparisons were based on the signal power which was determined as the root mean square across all MEG channels or all EEG channels over the left hemisphere. Statistical analyses were carried out using repeated measures univariate analyses of variance (ANOVA). Where appropriate, Greenhouse-Geisser adjustments were carried out.

Differences between experimental conditions were found starting at about $150 \mathrm{~ms}$ after stimulus onset. This is illustrated in Fig. 1 for the MEG data and in Fig. 2 for the EEG data.

Fig. 1 shows the grand averaged MEG waveforms for all experimental conditions. The lower graph demonstrates the grand average rms-waveforms; the upper graph presents the grand average for two of the 37 magnetic channels. The chosen channels represent the minimum and maximum magnetic field strength at the M180 component in each subject. After the early components a slow component lasting up to $700 \mathrm{~ms}$ after stimulus onset is seen under all experimental conditions. For the integrated amplitude in the latency range from $350-550 \mathrm{~ms}$, which involves the M300/M400 complex, the 'false font' condition showed larger amplitudes than the 'word', 'shapes' and 'dots' conditions. ANOVA comparing the four Stimulus Classes revealed a significant main effect for the integrated rms amplitude $(F(3 / 36)=5.24 ; \epsilon=0.572 ; P<0.02)$.

Fig. 2 shows the grand average EEG waveforms of a subset of electrodes. (One subject had to be excluded from analysis of EEG data because the impedance of the reference electrode has increased during the measurement). Differential effects were also seen starting at $150 \mathrm{~ms}$ after stimulus onset. In contrast to the MEG data, the largest amplitudes (the absolute value) of the integrated amplitude from 350-550 ms are observed for the 'dots' condition, and the smallest for the 'word' condition. The two-way ANOVA for a Stimulus Classes $\times$ Electrodes design using the left hemispheric electrodes revealed statistically significant differences for the integrated amplitude from 350-550 ms. Significant main effects of Stimulus Class $(F(3,33)=6.54 ; \epsilon=0.726 ; \quad P<0.006)$ and Electrodes $(F(3,33)=96.27 ; \epsilon=0.696 ; P<0.001)$, as well as signifi-
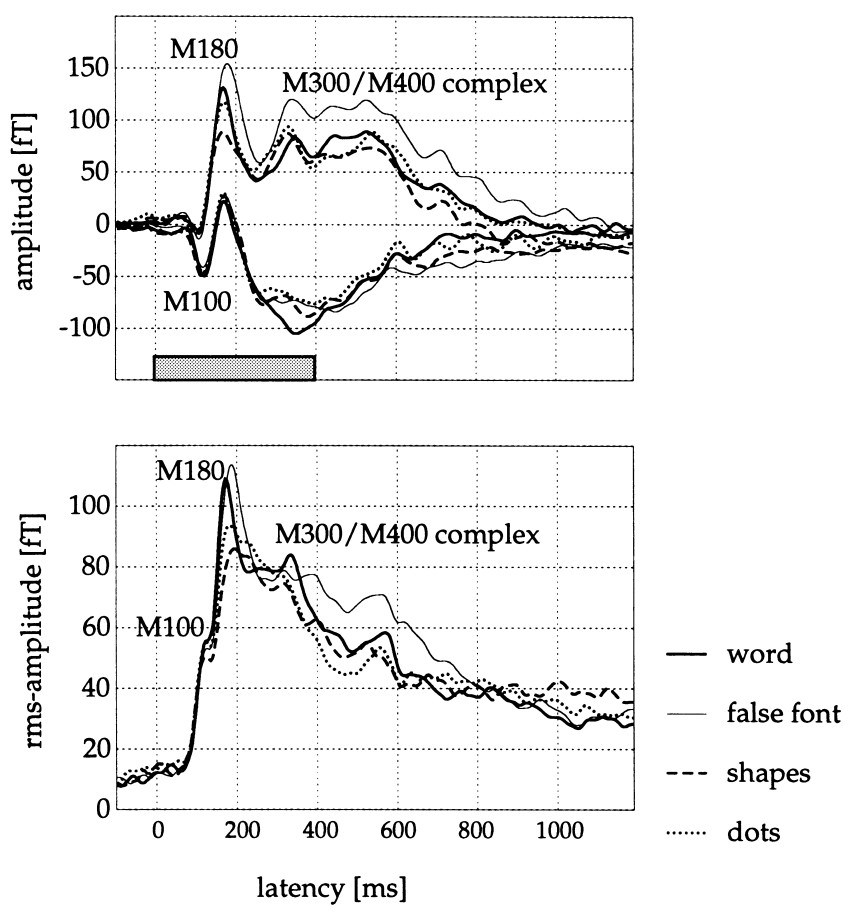

Fig. 1. Grand averaged MEG waveforms are shown separately for the different experimental conditions. The thick solid line codes the 'word' condition, the thin solid line the 'false font', the dashed line the 'shapes' and the dotted line the 'dots' condition. The lower part demonstrates the grand average rms waveforms recorded from the left hemisphere. The upper panel illustrates the grand average of two selected channels from the array of 37 magnetic channels. The channels were selected such that they represent the minimum and maximum magnetic field strength at the M180 component in each subject. Corresponding to this functional normalization, the positions varied across subjects with respect to their precise anatomical location on the scalp. The stimulus duration is indicated at the axis of the upper panel. 

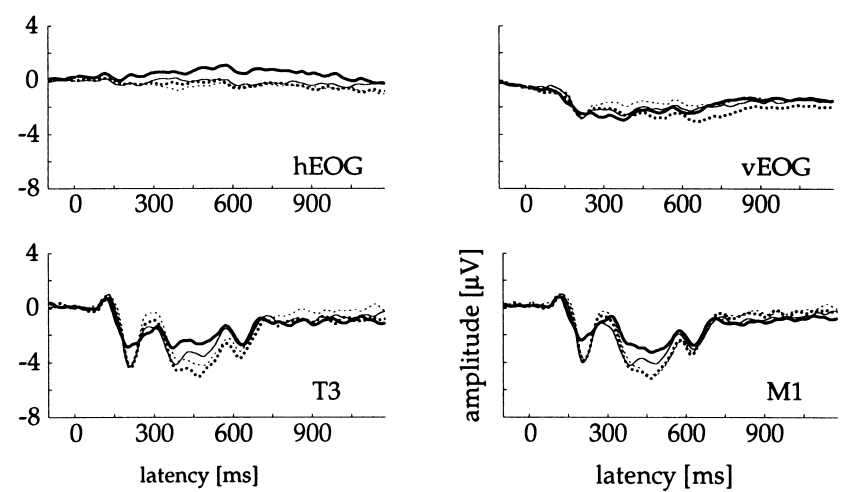

- word

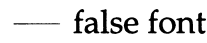

shapes dots

Fig. 2. Grand average EEG waveforms at the temporal and mastoidal electrodes over the left hemisphere (against $\mathrm{Cz}$ as a reference) as well as the horizontal and vertical electrooculogram are shown. Line styles are compatible to those in Fig. 1.

cant interactions of Stimulus Class $\times$ Electrodes $(F(9,99)=7.99 ; P<0.001 ;$ Wilk's Lambda: $R=21.30$; $P<0.02$ ) were found.

Both EEG and MEG data indicated significant differences between experimental conditions, but the ranking between conditions was different with the order of ranks not being simply mirrored. To shed further light on these discrepancies between EEG and MEG data for the latency range 350-550 ms, the signal power in the EEG data for this latency range was determined. Therefore, EEG data were rereferenced to average reference, and the root mean square was calculated for left hemispheric electrode positions. Both EEG and MEG rms values were transformed into $z$-scores. The mean $z$-scores and their SEs are shown in Fig. 3 which illustrates the different rankings between experimental conditions in EEG and MEG data. Across both measurement techniques the signal power was smallest for the 'word' condition.

The two-way ANOVA with four Stimulus Classes and two Measurement Techniques as repeated measures factors yielded a significant interaction Stimulus Class $\times$ Measurement Technique $(F(3,33)=15.12 ; P<0.001)$. The main effect of Stimulus Classes did not survive the Greenhouse-Geisser adjustment $(F(3,33)=3.11 ; \epsilon=0.603$; $P<0.07)$. However, multivariate analysis still indicated a significant difference between the conditions (Wilk's Lambda: $R=4.89 ; P<0.03$ ).

The combined comparison of the normalized power of EEG and MEG signals over the left hemisphere confirmed the different rankings of integrated amplitudes from 350 to $550 \mathrm{~ms}$ between conditions for EEG and MEG data as shown by the significant interaction. In addition, the main effect for Stimulus Classes indicated that the order of ranks was not simply mirrored.

These data reinforce the theoretical supposition that MEG and EEG may differentially image ongoing brain activity. The pattern of results indicates more activation of neurons in the gyri and, if in a sulcus, then across the complete structure, during the perception of 'shapes' and 'dots' stimuli, as compared to the perception of 'word' and 'false font' stimuli. (Activation in both opposing walls of the sulcus results in a radially oriented equivalent current flow [7]). The most plausible interpretation is that activated sources in the 'shapes' and 'dots' condition extend across larger areas than those in the 'false font' and 'word' condition. Source modeling of such an activational pattern will be hampered if restricted to the estimation of equivalent current dipoles. It might be more adequate to also model higher order terms in the multipole expansion as one possibility of accounting for the distribution of a source. Another possibility might be to model the sources as patches on the cortical surface [6].

It is clear from the amplitude results for the P300/N400 complex that MEG and EEG measured at the same latency interval deliver different effects, and, therefore, interpreting these two forms of data separately may result in misleading conclusions. Critical aspects to be considered are the extension and the multiplicity of the sources of event-related brain activity, the possibility of simultaneous activation of opposing walls of the sulci, as well as the locus of gyral (radial) activity which would affect the MEG signal to a lesser degree than EEG. In the present data several of these aspects were fulfilled which led to a different ranking of conditions for EEG and MEG data. In a previous study [2], in which MEG and EEG was recorded simultaneously during acoustic and phonetic processing which activated mainly the superior temporal plane, MEG and EEG measurements revealed similar differences and rankings of conditions.

Some of the present differences between EEG and MEG may be explained by a difference in the two methods that has not been adequately acknowledged in the literature: EEG and MEG are affected differently by averaging. If sources vary across trials and appear in different cerebral regions from trial to trial their impact on the event-related brain responses will be suppressed by averaging. However, this 'biological noise' is generally more strongly reduced for tangential sources than for radial ones. Sources in most of the sensory projection areas are primarily tangentially

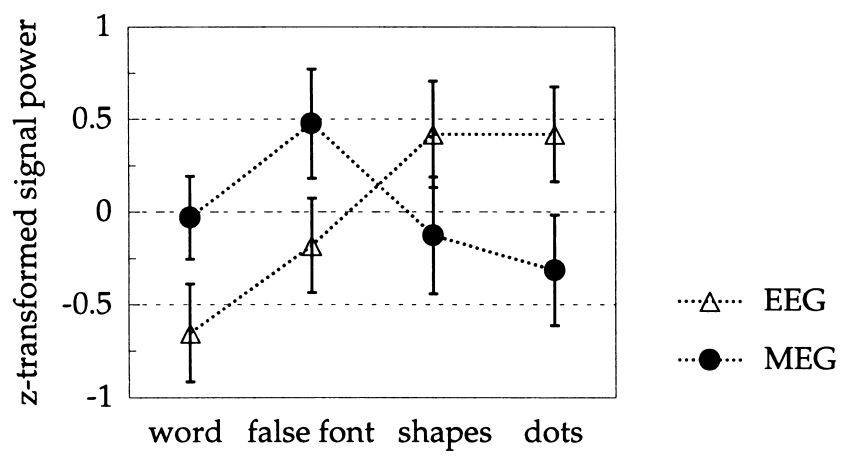

Fig. 3. Z-transformed values of the power of the EEG (empty triangles) and the MEG (filled circles) signal as well as their standard errors are demonstrated for all experimental conditions. Note the different rankings between experimental conditions in EEG and MEG data. 
oriented. Consequently, for such sources, typically activated early in the information processing, the signal-to-noise ratio is better for MEG measurements than for EEG measurements. In cases where the 'tangential' noise is strong, and sources are mainly radial, EEG might prove to be the more valuable source of information. The present result suggests that the two methods may deliver different information.

It can be summarized that the combined analysis of EEG and MEG data as supposed by many other authors [1,5] for purposes of source localizations is necessary to result in more realistic conclusions about the ongoing information processing. This is especially true if brain regions beyond the primary and secondary projection areas are activated. As both methods provide different information about the ongoing brain activity, their combined analysis is valuable, particularly for studies of higher order cognitive processing.

Research was supported by the Deutsche Forschungsgemeinschaft. We thank B. Maess, C. Pantev and M. Hoke for expert technical help and comments.

[1] Diekmann, V., Becker, W., Grözinger, B., Jürgens, R., Westphal, K.P. and Kornhuber, H.H., Localisation of focal epileptic activity with a simultaneous EEG and MEG model. In C. Baumgartner, L. Deecke, G. Stroink and S.J. Williamson (Eds.), Biomagnetism: Fundamental Research and Clinical Application, Vol. 7, Elsevier/IOS Press, Amsterdam, 1995, pp. 23-27.
[2] Eulitz, C., Diesch, E., Pantev, C., Hampson, S. and Elbert, T., Magnetic and electric brain activity evoked by the processing of tone and vowel stimuli, J. Neurosci., 15 (4 )(1995) 2748-2755.

[3] Eulitz, C., Maess, B., Pantev, C., Friederici, A., Feige, B. and Elbert, T., Oscillatory neuromagnetic activity induced by language and nonlanguage stimuli, Cogn. Brain Res., 4 (1996) 121-132.

[4] Frackowiak, R.S.J., Functional mapping of verbal memory and language, Trends Neurosci., 17 (1994) 109-115.

[5] Lütkenhöner, B., Möglichkeiten und Grenzen der neuromagnetischen Quellenanalyse, Lit Verlag, Münster, 1992, 137 pp.

[6] Lütkenhöner, B., Menninghaus, E., Steinsträter, O., Wienbruch, C., Gißler, M. and Elbert, T., Neuromagnetic source analysis using magnetic resonance images for the construction of source and volume conductor model, Brain Topogr., 7 (1995) 291-299.

[7] Lutzenberger, W., Elbert, T. and Rockstroh, B., A brief tutorial on the implications of volume conduction for the interpretation of the EEG, J. Psychophysiol., 1 (1987) 81-89.

[8] Pantev, C., Bertrand, O., Eulitz, C., Verkindt, C., Hampson, S., Schuirer, G. and Elbert, T., Specific tonotopic organizations of different areas of the human auditory cortex revealed by simultaneous magnetic and electric recordings, Electroenceph. clin. Neurophysiol., 94 (1995) 26-40.

[9] Williamson, J.S. and Kaufman, L., Theory of neuromagnetic fields. In F. Grandori, M. Hoke and G.L. Romani (Eds.), Auditory Evoked Magnetic Fields and Electric Potentials, Vol. 6, Advances in Audiology, Karger, Basel, 1990, pp. 1-39. 